\title{
Knowledge of Pelvic Floor Problems: A Study of Third Trimester, Primiparous Women
}

\author{
Aideen T O'Neill ${ }^{a}$, Joanne Hockey ${ }^{b}$, Patrick O’Brien ${ }^{c}$, Amanda Williams ${ }^{d}$, Tim P Morris ${ }^{e}$, \\ Tahira Khan ${ }^{f}$, Emma Hardwick ${ }^{b}$, Wai Yoong ${ }^{a}$ \\ a Department of Obstetrics and Gynaecology, North Middlesex University Hospital NHS Trust, Sterling Way, London N18 \\ 1QX, United Kingdom; b Department of Obstetrics and Gynaecology, Royal Free London NHS Trust, London NW3 2QG, \\ United Kingdom; c University College London Hospital and The Portland Hospital for Women and Children, 205 - 209 Great \\ Portland Street, London W1W 5AH, United Kingdom; d Department of Clinical, Educational and Health Psychology, \\ University College London, London WC1 6BT, United Kingdom; e Hub for Trials Methodology Research, MRC Clinical Trials \\ Unit, University College London, Aviation House, 125 Kingsway, London WC2B 6NH, United Kingdom; f UCL Medical School, \\ Gower Street, London WC1E 6BD, United Kingdom.
}

Correspondence: Dr AT O'Neill, Department of Obstetrics and Gynaecology, North Middlesex University Hospital NHS Trust, Sterling Way, London N18 1QX, United Kingdom. Tel: +44 208887 4083. Fax: 0208887 2732. Email aideen.oneill@nhs.net.

\section{Declaration of Potential Conflicts of Interest}

P. O'Brien is a member of the council of the Royal College of Obstetrics \& Gynaecology.

\section{Contribution to Authorship}

AT O’Neill: Protocol/ project development, data collection, data analysis, manuscript writing.

J Hockey: Protocol/ project development, manuscript editing.

P O'Brien: Protocol/ project development, manuscript editing.

A Williams: Protocol/ project development, manuscript editing.

TP Morris: Data Analysis, manuscript writing.

T Khan: Data Collection, manuscript editing

E Hardwick: Protocol/ project development, data collection.

W Yoong: Protocol/ project development, data analysis, manuscript writing.

\section{Word Count}

Abstract: 250

Manuscript text/ body: 1894 


\section{Abstract}

2 Introduction and Hypothesis: Pelvic floor problems in women (urinary incontinence, faecal

3 incontinence, uterovaginal prolapse) are common, and have an adverse effect on quality of

4 life. We hypothesised that there is low knowledge of these problems amongst primiparous 5 women in their third trimester of pregnancy.

6 Methods: We conducted a cross-sectional study in antenatal clinics of three hospitals in 7 London, United Kingdom, 2011 to 2013. Primiparous women, aged $\geq 18$ years and in the third 8 trimester of pregnancy, answered questions on pelvic floor problems. Knowledge scores

9 were calculated based on the proportion of questions answered correctly.

10 Results: 249 women completed the question set. The average knowledge score, across all 11 domains, was low at 45\% (confidence intervals included below). Scores were lowest for the 12 less common problems of faecal incontinence (35\%) and prolapse (36\%). The score for 13 urinary incontinence was higher at $63 \%$, but low when questions explored more detailed 14 levels of knowledge (41\%). Knowledge scores were positively-associated with both education 15 to tertiary level, and books as the information source on pregnancy and delivery. Only $35 \%$ of 16 women cited antenatal classes as a source.

17 Conclusions: Knowledge surrounding pelvic floor problems is low amongst third-trimester, 18 primiparous women in this London-based population. Adequate knowledge of these 19 problems is important in women being able to make informed choices about their antenatal 20 care and to seek help if problems arise. The data suggest scope for healthcare professionals 21 to raise these issues early during pregnancy, and to help women to access accurate sources 22 of information.

\section{Keywords}

24 Knowledge, pelvic floor, incontinence, prolapse, primiparous. 


\section{Brief Summary}

26 This cross-sectional study found that knowledge surrounding pelvic floor problems was low

27 amongst primiparous women in their third trimester of pregnancy.

\section{Abbreviations}

29 US: United States

30 UK: United Kingdom

31 UCL: University College London

32 NHS: National Health Service

33 IMD: Index of multiple deprivation

$34 \mathrm{Cl}$ : Confidence interval

35 
37 Pelvic floor problems amongst women are now recognised as an issue of significance. 38 Prevalence of urinary incontinence, faecal incontinence and uterovaginal prolapse have been 39 reported as $5 \%$ (6 months post-partum, experienced once or more per day) ${ }^{1} ; 4 \%$ (persisting 6 years post-partum) ${ }^{2}$; and $3.6 \%$ (of severity requiring surgery) $^{3}$, respectively. Symptoms have

41 been found to have an adverse affect on women's quality of life ${ }^{4,5,6,7}$. Aetiological factors include pregnancy and delivery ${ }^{8,9,10,11}$ and certain potential protective measures have been identified, including pelvic floor exercises and restricted weight gain ${ }^{12,13,14,15}$. There is recognition, too, of the fact that adequate knowledge of these problems is important in women being able to make informed choices about their antenatal care, including engagement in potential protective measures, and seeking help if problems arise $e^{16,17,18,19}$.

47 Two studies ${ }^{20,21}$ have quantitatively assessed knowledge levels amongst peri-partum women. 48 Both were conducted in the United States (US) and surveyed women after delivery. These 49 studies found that many women were not provided with information on pelvic floor problems 50 before delivery, and felt ill-prepared to deal with these issues.

51 Our study sought to quantify knowledge of pelvic floor problems amongst women in a 52 London-based population. We included only primiparous women in order to exclude 53 knowledge gained through experience, and only those in the third trimester (the point by 54 which one would expect women to have had access to information on these problems). We hypothesized that knowledge levels would be low. We also sought to identify factors

56 associated with higher knowledge levels, and the information sources used by women, this with a view to informing potential strategies to address knowledge shortfalls.

\section{$58 \quad$ Materials and Methods}

59 The study was undertaken from May 2011 to December 2013 at antenatal clinics of three centres in London, United Kingdom (UK):

61 (1) The Royal Free London NHS Trust (the Royal Free), a hospital funded by the national 
health service (NHS) (at which care is free at the point of use), and a campus of University College London (UCL) Medical School.

(2) The North Middlesex University Hospital Trust (the North Middlesex), also an NHSfunded hospital and campus of UCL Medical School.

(3) The Harley Street Centre for Women, a clinic of The Portland Hospital for Women and Children (the Portland Hospital) which is owned and operated by the private healthcare company, HCA International, and at which care is directly paid for by individuals (or their insurance company) using the service.

Women attending these clinics, who fulfilled the criteria of being primiparous, in the third trimester and aged 18 years or above, were invited to complete a set of 21 questions. These were self-administered. The questions were designed to elicit respondents' knowledge on the subjects of urinary incontinence, faecal incontinence and prolapse, including how they relate to pregnancy and childbirth. The question set is presented in Supporting Information S1. Examples of questions (to which answers were Agree/ Disagree/ Don't Know), included: "Being pregnant or giving birth may lead to urine leakage"; "Certain exercises can be done to reduce development of urine leakage"; "Pelvic organ prolapse is more common in thin women than in overweight women"; and "Leakage of stool only occurs in older women". Within the urinary incontinence section of the question set, certain questions were structured to distinguish detailed from superficial knowledge: "Whether exercises are done before birth, or instead after birth, does not affect how well they work"; and "If urine leakage happens related to pregnancy or birth, it is short-term in duration". Further questions explored the information sources that respondents had used in preparing for pregnancy and delivery and elicited demographic characteristics. In formulating the questions, we made reference to those developed in previous relevant research ${ }^{20}, \underline{21}, 22$, and were assisted by academic psychology at UCL. The question set was made available in four languages (English, Turkish, Somalian and Arabic). All women who agreed to participate signed a consent form after reviewing an information sheet and having had an opportunity to ask questions. 
91 the answer. Scores were calculated for each of the domains of urinary incontinence, faecal

92 incontinence and uterovaginal prolapse individually, and in aggregate across all domains

93 (resulting in a composite score for each woman).

94 The following demographic factors were assessed:

95 - Educational score: Calculated for each respondent based on level of formal education:

96 Degree (3 points), A-levels (school exams in England, typically taken aged 17 to 18) or 97 diploma (2 points), and GCSEs (school exams in England, typically taken aged 14 to 98 16) (1 point).

99 - Index of multiple deprivation (IMD): Ascertained for each respondent based on her 100 residential postcode. IMD is an index, compiled by the UK's Department for 101 Communities and Local Government, measured for small geographical areas in 102 England, which allows relative comparison of deprivation of geographical areas based 103 on seven distinct dimensions including income, employment, health deprivation and 104 disability, education skills and training, barriers to housing and services, crime and $105 \quad$ living environment ${ }^{23}$.

106 - Maternal age

107 - Percentage of life spent in the UK

108 Ethnicity

109 Statistical Analysis

110 Characteristics of participating women were summarised as a frequency for categorical data,

111 mean \pm standard deviation for continuous variables, and median (quartiles) for the percentage

112 of life lived in the UK. Multivariable linear regression was used to estimate the associations 113 between knowledge levels, background characteristics and knowledge sources used. Further 114 details of the statistical methods used are summarised in Supporting Information S2.

\section{Ethical Approval}

116 The study was approved by the UK Health Research Authority framework (Oxfordshire 117 Research Ethics Committee C, 25/03/2011), and by research and development committees at 118 the individual centres. 


\section{Results}

120249 women completed the question set, over a total of 72 clinics: 211 from the two NHS-

121 funded hospitals and 38 from the privately-funded hospital. Table 1 summarises the

122 demographics of respondents by centre. Mean gestational age was $33 \pm 5$ weeks. Based on

123 data from the first 20 clinics (the proportion at which percentage participation was recorded),

124 an average of $15 \%$ of clinic attendees were eligible to participate, and of those, an average of

$12595 \%$ agreed to participate.

126 The average composite knowledge score (aggregated across all domains and all centres), 127 was $45 \%$ out of a maximum of $100 \%$ (95\% confidence interval (Cl) $42-48 \%)$. Scores were 128 lowest in the domains of faecal incontinence: $35 \%(95 \% \mathrm{Cl} 32-39 \%)$, and uterovaginal 129 prolapse: $36 \%(95 \% \mathrm{Cl} 33-40 \%)$, and highest in the domain of urinary incontinence: $63 \%$ $130(60-66 \%)$. However, when the urinary incontinence score was calculated across the 131 discriminating questions (which were structured to distinguish detailed from superficial 132 knowledge), the score for urinary incontinence was comparable to the other domains at $41 \%$ $133(95 \% \mathrm{Cl} 36-47) \%$.

134 There was a positive association between knowledge scores and education to tertiary level, in 135 that knowledge scores decreased by $18 \%(95 \% \mathrm{Cl}-29 \%$ to $-7 \%)$ when education was to mid136 secondary school versus to tertiary/ degree level (Table 3). There was no significant 137 association between knowledge scores and any of maternal age, proportion of life spent in 138 the UK, ethnicity or deprivation index (Tables 3 and 4).

139 The most commonly cited information sources on pregnancy and delivery used by 140 participants were internet and books (84\% and $82 \%$ of participants, respectively) (Table 5). 141 Only $35 \%$ cited antenatal classes as a source of information. Books were the only 142 information source with a significant positive association with knowledge scores (scores 143 increasing by $+10 \%$ if books were cited as a source) $(95 \% \mathrm{Cl} 2-18 \%)$. 
144 There was a small, statistically significant difference between knowledge scores at one of the 145 centres (The North Middlesex) versus the other two centres (the Portland and the Royal Free) 146 (Table 2, $\mathrm{P}=0.02)$.

\section{Discussion}

148 In this cross-sectional study of primiparous women in the third-trimester of pregnancy, as 149 hypothesized, knowledge of pelvic floor problems was found to be low. This finding of poor 150 knowledge levels is consistent with the results of previous US-based studies. However, our

151 study refined the approach used in those previous studies, by confining participation to 152 antenatal women only, based on the premise that it is antenatally-gained rather than 153 postnatally-gained knowledge that can optimise women's ability to make informed choices on 154 these matters.

155 Of all of the demographic factors investigated, only formal education to tertiary level was 156 found to have a statistically significant positive association with knowledge scores. When 157 sources of knowledge were explored, books were the only information source that had a 158 statistically significant positive-association with knowledge scores. There was no significant 159 association between knowledge scores and any of doctors, midwives or antenatal classes as 160 sources. Only $35 \%$ of women cited antenatal classes as a source of information on pelvic 161 floor problems.

162 There was no association between knowledge scores and maternal age, proportion of life 163 spent in the UK or ethnicity.

164 There was a statistically significant difference $(p=0.02)$ between knowledge scores at one of 165 the centres (the North Middlesex, mean knowledge score 39\%) versus the other two centres 166 (Royal Free and Portland, mean knowledge scores $48 \%$ and $47 \%$, respectively). The authors 167 do not feel that there is a difference in the extent to which information on pelvic floor problems 168 is provided to women at the different centres. The latter view is consistent with the 169 observation made in the paragraph above regarding lack of association between knowledge 170 scores and doctors or midwives as the source of knowledge. It is interesting to note that 
171 Index of Multiple Deprivation is significantly higher (indicating more deprivation) $(\mathrm{p}<$

172 0.00001), at the North Middlesex versus at the other two centres (Table 1). If the analysis is

173 controlled for differences in IMD and education of participants by centre, the difference in

174 knowledge between centres is reduced such that it is no longer statistically significant $(p=$

175 0.44), although a residual difference does remain. We have not demonstrated a direct

176 association between knowledge scores and IMD (Table 3).

177 A central tenet of modern medical care is informed choice by patients. The low knowledge

178 levels identified in this study suggest that, with respect to pelvic floor problems, women are

179 not well-positioned to make informed choices about relevant aspects of their antenatal care,

180 including engagement in potential protective measures, nor to seek help if problems do arise.

181 The low scores suggest scope for education on pelvic floor problems. The data point to

182 potential specific areas for improvement. One such example is the lack of positive

183 association between knowledge levels and the most popular information source - the internet

184 - suggesting room for improvement of online content. This is in contrast to the positive

185 association with books as the source. Another example is the data illustrating the infrequency

186 with which antenatal classes are cited by women as information sources, and lack of positive

187 association of knowledge scores with any of antenatal classes, doctors and midwives. These

188 data suggest scope for healthcare professionals to raise these issues early in pregnancy, and

189 to help women to access accurate sources of information.

190 The main limitation of this study is the small size of some of the subpopulations identified,

191 specifically women using some of less popular knowledge sources. This limits the statistical

192 precision of some subgroup analyses. However this is an observational study in which the

193 investigators could not control the use of particular knowledge sources, and the proportion of

194 women citing particular sources is an important finding.

\section{Funding}

196 The study received no external funding. 


\section{Acknowledgements}

198 The authors would like to thank the women who participated in the study. We would also like 199 to thank Professor Allan Maclean, Emeritus Professor at University College London, who 200 provided on-going advice on the project and Professor Judith Stephenson, Margaret Pyke 201 Professor of Sexual \& Reproductive Health at University College London, who externally 202 reviewed the study methodology. We are grateful to University College London for their 203 sponsorship of the study. Finally we would like to thank the students and administrators who 204 assisted with data gathering, and who are listed in Supporting Information S3. 
206 Additional Supporting Information may be found in the online version of this article:

207 S1: Excerpts from Study Question Set.

208 S2: Statistical Methods.

209 S3: Students and Administrators who Assisted With Data Gathering

1 Wesnes SL, Hunskaar S, Bo K, Rortveit G. The effect of urinary incontinence status during pregnancy and delivery mode on incontinence postpartum. A cohort study. BJOG. 2009; Apr; 116(5):700-7.

2 Macarthur C, Wilson D, Hebison $\mathrm{P}$ et al. Faecal incontinence persisting after childbirth: a 12 year longitudinal study. BJOG. 2013; Jan;120(2):169-78.

3 Fritel X, Varnoux N, Zins $M$ et al. Symptomatic pelvic organ prolapse at midlife, quality of life, and risk factors. Obstet Gynecol. 2009; Mar; 113(3):609-16.

4 Kumar R1, Ooi C, Nicoll A et al. Anal incontinence and quality of life following obstetric anal sphincter injury. Arch Gynecol Obstet. 2012; Mar;285(3):591-7.

5 Lo J, Osterweil P, Li H et al. Quality of life in women with postpartum anal incontinence. Obstet Gynecol. 2010; Apr;115(4):809-14.

6 Lal M, Pattison HM, Allan TF, Callender R. Postcesarean pelvic floor dysfunction contributes to undisclosed psychosocial morbidity J Reprod Med. 2009; Feb;54(2):53-60.

7 Tucker J, Clifton V, Wilson A. Teetering near the edge; women's experiences of anal incontinence following obstetric anal sphincter injury: an interpretive phenomenological research study. Aust N Z J Obstet Gynaecol. 2014; Aug; 54(4):377-81.

8 Nygaard I, Barber MD, Burgio KL et al. Prevalence of symptomatic pelvic floor disorders in US women. JAMA. 2008; Sep 17;300(11):1311-6.

9 Quiroz LH1, Muñoz A, Shippey SH et al. Vaginal parity and pelvic organ prolapse. J Reprod Med. 2010; Mar-Apr;55(3-4):93-8.

10 Leijonhufvud A1, Lundholm $\mathrm{C}$ et al. Risks of stress urinary incontinence and pelvic organ prolapse surgery in relation to mode of childbirth. Am J Obstet Gynecol. 2011; Jan;204(1):70.

11 Gyhagen M, Bullarbo M, Nielsen TF. Faecal incontinence 20 years after one birth: a comparison between vaginal delivery and caesarean section. Int Urogynecol J. 2014; Oct;25(10):1411-8.

12 Hagen S, Stark D, Glazener C et al; POPPY Trial Collaborators. Individualised pelvic floor muscle training in women with pelvic organ prolapse (POPPY): a multicentre randomised controlled trial. Lancet. 2014. Mar 1;383(9919):796-806.

13 Boyle R1, Hay-Smith EJ, Cody JD et al. Pelvic floor muscle training for prevention and treatment of urinary and faecal incontinence in antenatal and postnatal women. Cochrane Database Syst Rev. 2012; Oct 17;10:CD007471.

14 Reilly ET1, Freeman RM, Waterfield MR et al. Prevention of postpartum stress incontinence in primigravidae with increased bladder neck mobility: a randomised controlled trial of antenatal pelvic floor exercises. BJOG. 2014 Dec;121 Suppl 7:58-66.

15 Chen $\mathrm{Y}$, Johnson B et al. The effect of body mass index on pelvic floor support 1 year postpartum. Reprod Sci. 2016 Feb;23(2):234-8.

16 Griffiths F, Pepper J, Jørstad-Stein EC et al. Group versus individual sessions delivered by a physiotherapist for female urinary incontinence: an interview study with women attending group sessions nested within a randomised controlled trial. BMC Womens Health. 2009; Sep 10;9:25.

17 Gyhagen M, Milsom I. Authors' reply: A comparison of the long-term consequences of vaginal delivery versus caesarean section of the prevalence, severity and bothersomeness of urinary incontinence subtypes: a national cohort study in primiparous women. BJOG. 2014 Aug;121(9):1176.

18 Wilson D, Dornan J, Milsom I, et al. UR-CHOICE: can we provide mothers-to-be with information about the risk of future pelvic floor dysfunction? Int Urogynecol J. 2014; Nov;25(11):1449-52.

19 O'Boyle AL, Davis GD, Calhoun BC. Informed consent and birth: protecting the pelvic floor and ourselves. Am J Obstet Gynecol. 2002; Oct;187(4):981-3.

20 McLennan MT, Melick CF, Alten B et al. Patients' knowledge of potential pelvic floor changes associated with pregnancy and delivery. Int Urogynecol J Pelvic Floor Dysfunct. 2006; Jan;17(1):226.

21 Howell EA. Lack of patient preparation for the postpartum period and patients' satisfaction with their obstetric clinicians. Obstet Gynecol. 2010; Feb;115(2 Pt 1):284-9.

22 Shah AD, Massagli MP, Kohli N et al. A reliable, valid instrument to assess patient knowledge 
about urinary incontinence and pelvic organ prolapse. Int Urogynecol J Pelvic Floor Dysfunct. 2008; Sep;19(9):1283-9.

23 Department for Communities and Local Government. (2011). English indices of deprivation 2010. Available: https://www.gov.uk/government/statistics/english-indices-of-deprivation-2010. Last accessed 14 Oct 2014. 